\title{
Pointwise characteristic factors for the multiterm return times theorem
}

\author{
Idris Assani†, Kimberly Presser $\ddagger$ \\ † Department of Mathematics, UNC Chapel Hill, NC 27599, \\ (e-mail:assani@math.unc.edu) \\ $\ddagger$ Department of Mathematics, Shippensburg University, PA 27517 \\ (e-mail:kjpres@ship.edu)
}

(Received December 30, 2010)

\begin{abstract}
This paper is an update and extension of a result the authors first proved in 2003. The goal of this paper is to study factors which are known to be $L^{2}$ characteristic for certain nonconventional averages and prove that these factors are pointwise characteristic for the multiterm return times averages.
\end{abstract}

In memory of Dan Rudolph. 


\section{Introduction}

A major result in ergodic theory in the late 1980's was the proof of the return times theorem by J. Bourgain [8] (which was later simplified by J. Bourgain, H. Furstenberg, Y. Katznelson, D. Ornstein in [9]). This theorem created a key strengthening of the Birkhoff's Pointwise Ergodic Theorem [7].

THEOREM 1. Let $(X, \mathcal{F}, \mu, T)$ be an ergodic dynamical system of finite measure and $f \in L^{\infty}(\mu)$. Then there exists a set $X_{f} \subset X$ of full measure such that for any other ergodic dynamical system $(Y, \mathcal{G}, \nu, S)$ with $\nu(Y)<\infty$ and any $g \in L^{\infty}(\nu)$ :

$$
\frac{1}{N} \sum_{n=1}^{N} f\left(T^{n} x\right) g\left(S^{n} y\right)
$$

converges $\nu$-a.e. for all $x \in X_{f}$.

Note that the set $X_{f}$ depends not only on the function $f$ chosen, but on the transformation $T$ in our dynamical system. In the BFKO proof [9] of the return times theorem, one of the keys to the argument was to decompose the given function using the Kronecker factor in order to prove the result independently for both the eigenfunctions and those functions in the orthocomplement of the Kronecker factor.

Using factors in convergence proofs in ergodic theory has long been a very useful tool. The notion of a characteristic factor is originally due to H. Furstenberg and is explicitly defined by H. Furstenberg and B. Weiss in [12].

Definition: When the limiting behavior of a non-conventional ergodic average for $(X, \mathcal{F}, \mu, T)$ can be reduced to that of a factor system $(Y, \mathcal{G}, \nu, T)$, we shall say that the latter is a characteristic factor of the former.

Prepared using etds.cls 
For each type of average under consideration, one will have to specify what is meant by reduced in the given case. In the case of H. Furstenberg and B. Weiss [12, they define the notion of characteristic factor for averages of the type

$$
\frac{1}{N} \sum_{n=1}^{N}\left(f \circ T^{n}\right)\left(g \circ T^{n^{2}}\right)
$$

Therefore their specific definition of characteristic factor is as follows.

Definition: If $\left\{p_{1}(n), p_{2}(n), \ldots, p_{k}(n)\right\}$ are $k$ integer-valued sequences, and $(Y, \mathcal{G}, \nu, T)$ is a factor of a system $(X, \mathcal{F}, \mu, T)$, we say that $\mathcal{G}$ is a characteristic factor for the scheme $\left\{p_{1}(n), p_{2}(n), \ldots, p_{k}(n)\right\}$, if for any $f_{1}, f_{2}, \ldots, f_{k} \in L^{\infty}(\mu)$ we have

$$
\frac{1}{N} \sum_{n=1}^{N}\left[f_{1} \circ T^{p_{1}(n)} \cdots f_{k} \circ T^{p_{k}(n)}-\mathbb{E}\left(f_{1} \mid \mathcal{G}\right) \circ T^{p_{1}(n)} \cdots \mathbb{E}\left(f_{k} \mid \mathcal{G}\right) \circ T^{p_{k}(n)}\right]
$$

converges to 0 in $L^{2}(\mu)$.

In 1998, D. Rudolph [17] extended the return times theorem to averages with more than two terms with his proof of the multiterm return times theorem. His proof answered one of the questions on the return times raised by I. Assant who proved the same result for weakly mixing systems in [1].

Theorem 2 (Multiterm Return Times Theorem) Let $k$ be any positive integer. For any ergodic dynamical system $(X, \mathcal{F}, T, \mu)$ and any $f \in L^{\infty}(\mu)$, there exists a set of full measure $X_{f}$ in $X$ such that if $x \in X_{f}$ for any other dynamical system $\left(Y_{1}, \mathcal{G}_{1}, S_{1}, \nu_{1}\right)$ and any $g_{1} \in L^{\infty}\left(\nu_{1}\right)$ there exists a set of full $\dagger$ These questions were brought up during D. Rudolph's visit to UNC-CH in 1991 while he was working on his joinings proof of Bourgain's return times theorem $\mathbf{1 5}$.

Prepared using etds.cls 
measure $Y_{g_{1}}$ in $Y_{1}$ such that if $y_{1} \in Y_{g_{1}}$ then ...for any other dynamical system $\left(Y_{k-1}, \mathcal{G}_{k-1}, S_{k-1}, \nu_{k-1}\right)$ and any $g_{k-1} \in L^{\infty}\left(\nu_{k-1}\right)$ there exists a set of full measure $Y_{g_{k-1}}$ in $Y_{k-1}$ such that if $y_{k-1} \in Y_{g_{k-1}}$ for any other dynamical system $\left(Y_{k}, \mathcal{G}_{k}, S_{k}, \nu_{k}\right)$ the average:

$$
\frac{1}{N} \sum_{n=1}^{N} f\left(T^{n} x\right) g_{1}\left(S_{1}^{n} y_{1}\right) g_{2}\left(S_{2}^{n} y_{2}\right) \cdots g_{k}\left(S_{k}^{n} y_{k}\right)
$$

converges $\nu_{k}$-a.e..

As we are interested in finding characteristic factors for ergodic dynamical systems this theorem is quoted here with the extra assumption of ergodicity for the dynamical system $(X, \mathcal{F}, T, \mu)$. D. Rudolph's proof of the multiterm return times theorem utilized the method of joinings and fully generic sequences. This led to an elegant proof of the theorem which avoided the study of the factor of the $\sigma$-algebra which was characteristic for the averages. So the higher order version of the Kronecker factor $\mathcal{K}$ which had been key to the BFKO [9] proof was not needed in D. Rudolph's argument. This paper seeks to determine what factors serve a role similar to the Kronecker factor $\mathcal{K}$ in this multiterm setting.

For our purposes we define the notion of pointwise characteristic factors for the multiterm return times averages as follows.

Definition: Consider $(X, \mathcal{F}, \mu, T)$ a measure preserving system. The factor $\mathcal{A}$ is pointwise characteristic for the $k$-th return times averages if for each $f \in L^{\infty}(\mu)$ we can find a set of full measure $X_{f}$ such that for each $x \in X_{f}$, for any other dynamical system $\left(Y_{1}, \mathcal{G}_{1}, S_{1}, \nu_{1}\right)$ and any $g_{1} \in L^{\infty}\left(\nu_{1}\right)$, there exists a set of full measure $Y_{g_{1}}$ such that for each $y_{1}$ in $Y_{g_{1}}$ then $\ldots$ for any other dynamical 
system $\left(Y_{k-1}, \mathcal{G}_{k-1}, S_{k-1}, \nu_{k-1}\right)$ and any $g_{k-1} \in L^{\infty}\left(\nu_{k-1}\right)$, there exist a set of full measure $Y_{g_{k-1}}$ in $Y_{k-1}$ such that if $y_{k-1} \in Y_{g_{k-1}}$ for any other dynamical system $\left(Y_{k}, \mathcal{G}_{k}, S_{k}, \nu_{k}\right)$ for $\nu_{k}$-a.e. $y_{k}$ the average

$$
\frac{1}{N} \sum_{n=1}^{N}\left[f\left(T^{n} x\right)-\mathbb{E}(f \mid \mathcal{A})\left(T^{n} x\right)\right] g_{1}\left(S_{1}^{n} y_{1}\right) g_{2}\left(S_{2}^{n} y_{2}\right) \cdots g_{k}\left(S_{k}^{n} y_{k}\right)
$$

converges to 0 .

In looking for potential characteristic factors for the general multiterm return times averages we consider the factors first used by H. Furstenberg to prove Szemerédi's Theorem [11. These factors are called $k$ step distal factors in [11. We denote these factors (which will be further defined in Section 2) as $\mathcal{A}_{k}$ using the notation from $\left[3\right.$ where these factors were shown to be $L^{2}$-characteristic for the averages

$$
\frac{1}{N} \sum_{n=1}^{N} \prod_{i=1}^{I} f_{i} \circ T^{i n}
$$

While the norm convergence of averages for $L^{2}$-characteristic factors can sometimes lead to pointwise characteristic properties, this is not always guaranteed to be the case. Thus it is of consequence to look at pointwise convergence in addition to investigating factors with respect to the norm convergence.

We will show that these $\mathcal{A}_{k}$ factors can be characterized in an inductive way by seminorms using Lemma 1.3 of [16]. Using these seminorms we will prove our first result

$\dagger$ This approach was used in two 2003 unpublished papers of the first author ([3] and what was ultimately combined into the published paper [4]). The first author thanks C. Demeter and N. Frantzikinakis for pointing out to him that the factors he defined with these seminorms were

Prepared using etds.cls 
THEOREM 3. The factors $\mathcal{A}_{k}$ are pointwise characteristic for the multiterm return times averages. More precisely, using the seminorms defining the $\mathcal{A}_{k}$ we can find pointwise uniform upper bounds of the multiterm return times averages.

The study of the nonconventional Furstenberg averages has seen important progress being made in the last seven years. In $\mathbf{1 3}$ and $\mathbf{1 8}$ the Host-Kra-Ziegler factors $\mathcal{Z}_{k}$ were created independently by B. Host, B. Kra and T. Ziegler and were shown to be characteristic in $L^{2}$ norm for the Furstenberg averages. Using these factors we prove our second result.

TheOREm 4. Let $(X, \mathcal{F}, \mu, T)$ be an ergodic measure preserving system. The HostKra-Ziegler factors $\mathcal{Z}_{k}$ are pointwise characteristic for the multiterm return times averages.

As the $\mathcal{Z}_{k}$ factors are smaller than the factors $\mathcal{A}_{k}$, and thus $\mathcal{A}_{k}^{\perp} \subseteq \mathcal{Z}_{k}^{\perp}$, the fact that the $\mathcal{A}_{k}$ factors are pointwise characteristic for the multiterm return times averages is a consequence of Theorem 4. But in our proof of Theorem 3 using the seminorm defining the factors $\mathcal{A}_{k}$ we obtain pointwise uniform upper bounds of the multiterm return times averages. With the $\mathcal{Z}_{k}$ factors we do not have such pointwise estimates. The uniform upper bounds are derived after integration combined with a limsup argument.

An unusual feature of our proof is that we use the previously established convergence result of $\mathrm{D}$. Rudolph and use that to prove that the factors we are in fact the ones introduced by H. Furstenberg in [11. A careful look at Theorem 10.2 in 11 indicates that the $\mathrm{k}$ step distal factors are $L^{2}$ characteristic for the Furstenberg averages.

Prepared using etds.cls 
studying are characteristic.

2. The k-step distal factors $\mathcal{A}_{k}$ factors are pointwise characteristic for the multiterm return times averages

Let $(X, \mathcal{F}, \mu, T)$ be an ergodic dynamical system on a probability measure space.

The factors $\mathcal{A}_{k}$ are defined in the following inductive way.

Definition:

- The factor $\mathcal{A}_{0}$ is equal to the trivial $\sigma$-algebra $\{X, \emptyset\}$

- For $k \geq 0$ the factor $\mathcal{A}_{k+1}$ is characterized by the following. A function $f \in \mathcal{A}_{k+1}^{\perp}$ if and only if

$$
N_{k+1}(f)^{4}:=\lim _{H} \frac{1}{H} \sum_{h=1}^{H}\left\|\mathbb{E}\left(f \cdot f \circ T^{h} \mid \mathcal{A}_{k}\right)\right\|_{2}^{2}=0
$$

Note that the factor $\mathcal{A}_{1}$ is the Kronecker factor of our ergodic transformation $T$ because

$$
N_{1}(f)^{4}=\lim _{H} \frac{1}{H} \sum_{h=1}^{H}\left\|\mathbb{E}\left(f \cdot f \circ T^{h} \mid \mathcal{A}_{0}\right)\right\|_{2}^{2}=\lim _{H} \frac{1}{H} \sum_{h=1}^{H}\left|\int f \cdot f \circ T^{h} d \mu\right|^{2} .
$$

In Lemma 1 we will verify that the $\mathcal{A}_{k}$ as defined above do actually form well-defined factors.

We will want to verify that these $\mathcal{A}_{k}$ are maximal isometric extensions. There are several equivalent ways of expressing this feature. We will be using the terminology found on pages 373-374 of [16] to specify how these factors form maximal isometric extensions. Furstenberg has shown in $\mathbf{1 0}$ that for any ergodic dynamical system $(X, \mathcal{F}, \mu, T)$ and any $T$-invariant factor $\mathcal{B}$ there is a unique maximal factor action

Prepared using etds.cls 
$\mathcal{K B} \subseteq \mathcal{F}$ which contains $\mathcal{B}$ so that in the Rohlin representation of $(T, \mathcal{K B})$, the space $\mathcal{Z}_{2}$ can be taken to be a compact metric space with isometric factor maps. This factor $\mathcal{K B}$ arises from the invariant algebras of the relatively independent joinings of $(X, \mathcal{F}, \mu, T)$ over the factor $\mathcal{B}$. This is precisely the notion of maximal isometric extension referred to in the next lemma.

Lemma 1. Let $(X, \mathcal{F}, \mu, T)$ be an ergodic dynamical system on a probability measure space. For $k \geq 2$ for each function $f \in L^{\infty}(\mu)$ the quantities $N_{k}(f)$ are well defined. Furthermore, they characterize factors of $T$ which are successive maximal isometric extensions.

Proof: Let us consider a general factor $\mathcal{A}$ of $T$ and $\mathbb{E}(\cdot \mid \mathcal{A})$ the projection onto this factor. The relatively independent joining of $T \times T$ over the factor $\mathcal{A}$ is the measure $\mu_{\mathcal{A}}$ defined for $f, g$ bounded functions as

$$
\int f \times g d \mu_{\mathcal{A}}:=\int \mathbb{E}(f \mid \mathcal{A}) \mathbb{E}(g \mid \mathcal{A}) d \mu
$$

By Birkhoff's ergodic theorem applied to $T \times T$ and the invariant measure $\mu_{\mathcal{A}}$ we have

$$
\begin{aligned}
\lim _{H} \frac{1}{H} \sum_{h=1}^{H}\left\|\mathbb{E}\left(f \cdot f \circ T^{h} \mid \mathcal{A}\right)\right\|_{2}^{2} & =\lim _{H} \frac{1}{H} \sum_{h=1}^{H} \int\left(f \cdot f \circ T^{h}\right)(x)\left(f \cdot f \circ T^{h}\right)(y) d \mu_{\mathcal{A}} \\
& =\left\|\mathbb{E}\left(f \times f \mid \mathcal{I}_{\mathcal{A}}\right)\right\|_{L^{2}\left(\mu_{\mathcal{A}}\right)}^{2}
\end{aligned}
$$

where $\mathcal{I}_{\mathcal{A}}$ is the $T \times T$ - $\mu_{\mathcal{A}}$ invariant $\sigma$-algebra.

If we denote by $N(f)$ the quantity

$$
N(f)^{4}=\lim _{H} \frac{1}{H} \sum_{h=1}^{H}\left\|\mathbb{E}\left(f \cdot f \circ T^{h} \mid \mathcal{A}\right)\right\|_{2}^{2}
$$

Prepared using etds.cls 
then Lemma 1.3 in $[\mathbf{1 6}$ tells us that $N(f)=0$ if and only if $\mathbb{E}(f \mid \mathcal{K} \mathcal{A})=0$ where $\mathcal{K} \mathcal{A}$ is the maximal isometric extension of $\mathcal{A}$.

Using these observations one can characterize the successive maximal isometric extensions. The trivial $\sigma$-algebra is $\mathcal{A}_{0}$. Then we define $\mathcal{A}_{1}=\mathcal{K} \mathcal{A}_{0}, \mathcal{A}_{2}=\mathcal{K} \mathcal{A}_{1}$ and more generally $\mathcal{A}_{k+1}=\mathcal{K} \mathcal{A}_{k}$. The seminorms characterizing these factors are well defined as $N_{k}(f)$ where

$$
N_{k}(f)^{4}=\lim _{H} \frac{1}{H} \sum_{h=1}^{H}\left\|\mathbb{E}\left(f \cdot f \circ T^{h} \mid \mathcal{A}_{k}\right)\right\|_{2}^{2} .
$$

In order to simplify the inductive parts of our argument, we first clarify the techniques that we will use in a series of small lemmas. This next lemma relies on an application of the spectral theorem which allows us to alternate between Wiener-Wintner and return times averages in our inductive argument.

LEMMA 2. Let $\left\{a_{n}\right\}$ be a sequence of complex numbers. If

$$
\sup _{N} \frac{1}{N} \sum_{n=1}^{N}\left|a_{n}\right|^{2}<\infty \text { and } \sup _{\epsilon}\left|\frac{1}{N} \sum_{1}^{N} a_{n} e^{2 \pi i n \epsilon}\right| \rightarrow 0
$$

then

$$
\frac{1}{N} \sum_{1}^{N} a_{n} g\left(S^{n} y\right) \rightarrow 0
$$

in $L^{2}(\nu)$ for all measure-preserving systems $(Y, \mathcal{G}, S, \nu)$.

Proof: This follows immediately from the proof of Theorem 3.1 in [2].

Next, we will use the following lemma which is an easy consequence of the Van der Corput lemma [14. It will help us simplify the Wiener-Wintner averages which will appear in the inductive argument.

Prepared using etds.cls 
LEMma 3. There exists an absolute constant $C$ such that for any $\left\{a_{n}\right\}$ bounded sequence of complex numbers and any positive integer $N$ we have

$$
\sup _{\epsilon}\left|\frac{1}{N} \sum_{n=1}^{N} a_{n} e^{2 \pi i n \epsilon}\right|^{2} \leq C\left(\frac{1}{H}+\frac{1}{H} \sum_{h=1}^{H}\left|\frac{1}{N} \sum_{n=1}^{N-h} a_{n} \overline{a_{n+h}}\right|\right)
$$

for $1 \leq H \leq N$

The following lemma will be useful in establishing the basis step of our next theorem. It gives a pointwise upper bound for the return times averages for two terms, case studied in the BFKO 9 paper.

Lemma 4. Let $(X, \mathcal{F}, \mu, T)$ be an ergodic dynamical system and $f \in L^{\infty}(\mu)$. Let us denote by $\mathcal{K}_{T}$ its Kronecker factor. Then there exists a universal set $X_{f}$ depending only on $f$ and the system $(X, \mathcal{F}, \mu, T)$ such that for any dynamical system $\left(Y_{1}, \mathcal{G}_{1}, S_{1}, \nu_{1}\right)$ and any $g_{1} \in L^{\infty}\left(\nu_{1}\right)$ we have

$$
\underset{N}{\limsup }\left|\frac{1}{N} \sum_{n=1}^{N} f\left(T^{n} x\right) g_{1}\left(S_{1}^{n} y\right)\right| \leq\left\|\mathbb{E}\left(f \mid \mathcal{K}_{T}\right)\right\|_{2}\left\|g_{1}\right\|_{\infty}
$$

Proof: By the BFKO return times theorem, we know that the Kronecker factor is pointwise characteristic. So we have on a universal set $X_{f}$ of full measure

$$
\underset{N}{\limsup }\left|\frac{1}{N} \sum_{n=1}^{N} f\left(T^{n} x\right) g_{1}\left(S_{1}^{n} y_{1}\right)\right|=\underset{N}{\limsup }\left|\frac{1}{N} \sum_{n=1}^{N} \mathbb{E}\left(f \mid \mathcal{K}_{T}\right)\left(T^{n} x\right) g_{1}\left(S_{1}^{n} y_{1}\right)\right|
$$

$\nu_{1}$ a.e., for any dynamical system $\left(Y_{1}, \mathcal{G}_{1}, S_{1}, \nu_{1}\right)$ and any $g_{1} \in L^{\infty}\left(\nu_{1}\right)$.

Therefore for any dynamical system $\left(Y_{1}, \mathcal{G}_{1}, S_{1}, \nu_{1}\right)$ and any $g_{1} \in L^{\infty}\left(\nu_{1}\right)$,

$$
\underset{N}{\limsup }\left|\frac{1}{N} \sum_{n=1}^{N} f\left(T^{n} x\right) g_{1}\left(S_{1}^{n} y_{1}\right)\right| \leq \underset{N}{\limsup }\left|\frac{1}{N} \sum_{n=1}^{N} \mathbb{E}\left(f \mid \mathcal{K}_{T}\right)\left(T^{n} x\right)\right|\left\|g_{1}\right\|_{\infty}
$$

Using the Cauchy Schwartz inequality we have then

$$
\underset{N}{\limsup }\left|\frac{1}{N} \sum_{n=1}^{N} f\left(T^{n} x\right) g_{1}\left(S_{1}^{n} y_{1}\right)\right| \leq \underset{N}{\limsup }\left(\frac{1}{N} \sum_{n=1}^{N} \mid\left(\left.\mathbb{E}\left(f \mid \mathcal{K}_{T}\right)\left(T^{n} x\right)\right|^{2}\right)^{1 / 2}\left\|g_{1}\right\|_{\infty} .\right.
$$

Prepared using etds.cls 
Then by applying Birkhoff's pointwise ergodic theorem we have

$$
\underset{N}{\limsup }\left|\frac{1}{N} \sum_{n=1}^{N} f\left(T^{n} x\right) g_{1}\left(S_{1}^{n} y_{1}\right)\right| \leq\left(\int \mid\left(\left.\mathbb{E}\left(f \mid \mathcal{K}_{T}\right)\right|^{2} d \mu\right)^{1 / 2}\left\|g_{1}\right\|_{\infty},\right.
$$

which is the upper bound announced in this lemma.

We will prove our first main result, Theorem 3, in the course of proving the following more detailed statement.

Theorem 5. Let $k$ be any positive integer. For any ergodic dynamical system $(X, \mathcal{F}, \mu, T)$ and for each $f \in L^{\infty}(\mu)$ we can find a set of full measure $X_{f}$ such that for each $x \in X_{f}$, for any other dynamical system $\left(Y_{1}, \mathcal{G}_{1}, S_{1}, \nu_{1}\right)$ and any $g_{1} \in L^{\infty}\left(\nu_{1}\right)$ with $\left\|g_{1}\right\|_{\infty} \leq 1$, there exists a set of full measure $Y_{g_{1}}$ such that for each $y_{1}$ in $Y_{g_{1}}$ then $\ldots$ for any other dynamical system $\left(Y_{k-1}, \mathcal{G}_{k-1}, S_{k-1}, \nu_{k-1}\right)$ and any $g_{k-1} \in L^{\infty}\left(\nu_{k-1}\right)$ with $\left\|g_{k}\right\|_{\infty} \leq 1$ there exist a set of full measure $Y_{g_{k-1}}$ in $Y_{k-1}$ such that if $y_{k-1} \in Y_{g_{k-1}}$ for any other dynamical system $\left(Y_{k}, \mathcal{G}_{k}, S_{k}, \nu_{k}\right)$ for $\nu_{k}$-a.e. $y_{k}$

- the average

$$
\frac{1}{N} \sum_{n=1}^{N}\left[f\left(T^{n} x\right)-\mathbb{E}\left(f \mid \mathcal{A}_{k}\right)\left(T^{n} x\right)\right] g_{1}\left(S_{1}^{n} y_{1}\right) g_{2}\left(S_{2}^{n} y_{2}\right) \cdots g_{k}\left(S_{k}^{n} y_{k}\right)
$$

converges to 0 .

- Thus for $f \in \mathcal{A}_{k}^{\perp}$ the average

$$
\frac{1}{N} \sum_{n=1}^{N} f\left(T^{n} x\right) g_{1}\left(S_{1}^{n} y_{1}\right) g_{2}\left(S_{2}^{n} y_{2}\right) \cdots g_{k}\left(S_{k}^{n} y_{k}\right)
$$

converges to $0 \nu_{k}$-a.e..

Prepared using etds.cls 
- Also we have the following pointwise upper bound for our limit

$$
\underset{N}{\limsup }\left|\frac{1}{N} \sum_{n=1}^{N} f\left(T^{n} x\right) g_{1}\left(S_{1}^{n} y\right) g_{2}\left(S_{2}^{n} y_{2}\right) \cdots g_{k}\left(S_{k}^{n} y_{k}\right)\right|^{2} \leq C N_{k+1}(f)^{2}
$$

Proof: The basis step for the induction of the statement in (2) was done in the BFKO 9 proof of Bourgain's Return Times Theorem . Here it was shown that $\mathcal{A}_{1}=\mathcal{K}_{T}$ was pointwise characteristic for averages of the type

$$
\frac{1}{N} \sum_{n=1}^{N} f\left(T^{n} x\right) g_{1}\left(S_{1}^{n} y_{1}\right)
$$

In Lemma 4. we showed that the quantity $\left\|\mathbb{E}\left(f \mid \mathcal{A}_{1}\right)\right\|_{2}\left\|g_{1}\right\|_{\infty}$ is a pointwise upper bound for the limsup of the absolute value of the averages where $\mathcal{K}_{S_{1}}$ is the Kronecker factor for $S_{1}$. This last term is itself less than $\left\|\mathbb{E}\left(f \mid \mathcal{A}_{1}\right)\right\|_{2}$ because $\left\|g_{1}\right\|_{\infty} \leq 1$. Thus we have reached the inequality

$$
\underset{N}{\limsup }\left|\frac{1}{N} \sum_{n=1}^{N} f\left(T^{n} x\right) g_{1}\left(S_{1}^{n} y\right)\right|^{2} \leq\left\|\mathbb{E}\left(f \mid \mathcal{A}_{1}\right)\right\|_{2}^{2}
$$

We want to get a better upper bound namely $C N_{2}(f)$ where $C$ is an absolute constant. To this end we apply the Van der Corput lemma to obtain

$$
\begin{gathered}
\limsup _{N}\left|\frac{1}{N} \sum_{n=1}^{N} f\left(T^{n} x\right) g_{1}\left(S_{1}^{n} y\right)\right|^{2} \leq \\
\underset{N}{\limsup C}\left[\frac{1}{H}+\left(\frac{1}{H} \sum_{h=1}^{H}\left|\frac{1}{N} \sum_{n=1}^{N-h} f\left(T^{n} x\right) f\left(T^{n+h} x\right) g_{1}\left(S_{1}^{n} y\right) g_{1}\left(S_{1}^{n+h} y_{1}\right)\right|\right)\right] \leq \\
C\left[\frac{1}{H}+\left(\frac{1}{H} \sum_{h=1}^{H} \limsup _{N}\left|\frac{1}{N} \sum_{n=1}^{N-h} f\left(T^{n} x\right) f\left(T^{n+h} x\right) g_{1}\left(S_{1}^{n} y\right) g_{1}\left(S_{1}^{n+h} y_{1}\right)\right|\right)\right]
\end{gathered}
$$

Applying the inequality (5) to each of the functions $f \cdot f \circ T^{h}$ yields

$$
\underset{N}{\limsup }\left|\frac{1}{N} \sum_{n=1}^{N} f\left(T^{n} x\right) g\left(S^{n} y\right)\right|^{2} \leq C\left[\frac{1}{H}+\left(\frac{1}{H} \sum_{h=1}^{H}\left\|\mathbb{E}\left(f \cdot f \circ T^{h} \mid \mathcal{A}_{1}\right)\right\|_{2}^{2}\right)^{1 / 2}\right]
$$

Prepared using etds.cls 
By taking the limit with $H$ we get the better estimate

$$
\underset{N}{\limsup }\left|\frac{1}{N} \sum_{n=1}^{N} f\left(T^{n} x\right) g\left(S^{n} y\right)\right|^{2} \leq C N_{2}(f)^{2} .
$$

which shows clearly that $\mathcal{A}_{1}$ satisfies (3) in the basis step.

Assume that for any $f \in L^{\infty}(\mu)$ and $1 \leq j<k$ we can find sets $X_{f}$ of full measure such that if $x \in X_{f}$, then for any other dynamical system $\left(Y_{1}, \mathcal{G}_{1}, S_{1}, \nu_{1}\right)$ and any $g_{1} \in L^{\infty}\left(\nu_{1}\right)$ with $\left\|g_{1}\right\|_{\infty} \leq 1$, there exists a set of full measure $Y_{g_{1}}$ such that for each $y_{1}$ in $Y_{g_{1}}$ then $\ldots$ for any other dynamical system $\left(Y_{j-1}, \mathcal{G}_{j-1}, S_{j-1}, \nu_{j-1}\right)$ and any $g_{j-1} \in L^{\infty}\left(\nu_{j-1}\right)$ with $\left\|g_{j-1}\right\|_{\infty} \leq 1$ there exist a set of full measure $Y_{g_{j-1}}$ in $Y_{j-1}$ such that if $y_{j-1} \in Y_{g_{j-1}}$ for any other dynamical system $\left(Y_{j}, \mathcal{G}_{j}, S_{j}, \nu_{j}\right)$ and any $g_{j} \in L^{\infty}\left(\nu_{j}\right)$ with $\left\|g_{j}\right\|_{\infty} \leq 1$ for $\nu_{j}$-a.e. $y_{j}$ we have

- the average

$$
\frac{1}{N} \sum_{n=1}^{N}\left[f\left(T^{n} x\right)-\mathbb{E}\left(f \mid \mathcal{A}_{j}\right)\left(T^{n} x\right)\right] g_{1}\left(S_{1}^{n} y_{1}\right) \cdots g_{j}\left(S_{j}^{n} y_{j}\right)
$$

converges to 0 .

- Also we have the upper bound

$$
\underset{N}{\limsup }\left|\frac{1}{N} \sum_{n=1}^{N} f\left(T^{n} x\right) g_{1}\left(S_{1}^{n} y\right) g_{2}\left(S_{2}^{n} y_{2}\right) \cdots g_{j}\left(S_{j}^{n} y_{j}\right)\right|^{2} \leq C N_{j+1}(f)^{2}
$$

Lemma 5. Let $f$ be an element of $f \in L^{\infty}$ and let $g_{i}, S_{i}$ and $y_{i}$ be as defined in the preceding paragraph. If

$$
B_{N}=\sup _{\epsilon}\left|\frac{1}{N} \sum_{n=1}^{N} f\left(T^{n} x\right) g_{1}\left(S_{1}^{n} y_{1}\right) \cdots g_{k-1}\left(S_{k-1}^{n} y_{k-1}\right) e^{2 \pi i n \epsilon}\right|^{2}
$$

then

$$
\limsup _{N} B_{N} \leq C N_{k}(f)^{2}
$$

Prepared using etds.cls 
for some absolute constant $C$. Here the constant $C$ is independent of the $f, g_{i}, S_{i}$ and $y_{i}$.

Proof: By Lemma 3, there exists a constant $C$ such that for $1 \leq H \leq N$

$$
\begin{aligned}
B_{N} \leq & C\left(\frac{1}{H}+\frac{1}{H} \sum_{h=1}^{H} \mid \frac{1}{N} \sum_{n=1}^{N-h}\left(f \cdot \overline{f \circ T^{h}}\right)\left(T^{n} x\right)\right. \\
& \left.\cdot\left(g_{1} \cdot \overline{g_{1} \circ S_{1}^{h}}\right)\left(S_{1}^{n} y_{1}\right) \cdots\left(g_{k-1} \cdot \overline{g_{k-1} \circ S_{k-1}^{h}}\right)\left(S_{k-1}^{n} y_{k-1}\right) \mid\right) .
\end{aligned}
$$

From our inductive hypothesis, we know that for each $h$ there is a set of full measure $X_{f \cdot \overline{f \circ T^{h}}}$ on which

$$
\begin{aligned}
& \mid \frac{1}{N} \sum_{n=1}^{N-h}\left[\left(f \cdot \overline{f \circ T^{h}}\right)\left(T^{n} x\right)-\left(\mathbb{E}\left(f \cdot \overline{f \circ T^{h}} \mid \mathcal{A}_{k-1}\right)\left(T^{n} x\right)\right]\right. \\
& \cdot\left(g_{1} \cdot \overline{g_{1} \circ S_{1}^{h}}\right)\left(S_{1}^{n} y_{1}\right) \cdots\left(g_{k-1} \cdot \overline{g_{k-1} \circ S_{k-1}^{h}}\right)\left(S_{k-1}^{n} y_{k-1}\right) \mid \rightarrow 0 .
\end{aligned}
$$

Therefore, the intersection of these sets $X_{f \cdot \overline{f \circ T^{h}}}$ over $h$ gives a set of full measure $\widehat{X_{f}}$ on which

$$
\begin{aligned}
\underset{N}{\limsup } B_{N} \leq & \limsup _{N} C\left(\frac{1}{H}+\frac{1}{H} \sum_{h=1}^{H} \mid \frac{1}{N} \sum_{n=1}^{N-h}\left(\mathbb{E}\left(f \cdot \overline{f \circ T^{h}} \mid \mathcal{A}_{k-1}\right)\left(T^{n} x\right)\right.\right. \\
& \left.\cdot\left(g_{1} \cdot \overline{g_{1} \circ S_{1}^{h}}\right)\left(S_{1}^{n} y_{1}\right) \cdots\left(g_{k-1} \cdot \overline{g_{k-1} \circ S_{k-1}^{h}}\right)\left(S_{k-1}^{n} y_{k-1}\right) \mid\right)
\end{aligned}
$$

for all $H$.

The Cauchy-Schwartz inequality gives us

$$
\begin{aligned}
\underset{N}{\limsup B_{N} \leq} & \limsup _{N} C\left(\frac{1}{H}+\frac{1}{H} \sum_{h=1}^{H}\left(\frac{1}{N} \sum_{n=1}^{N-h} \mid\left(\left.\mathbb{E}\left(f \cdot \overline{f \circ T^{h}} \mid \mathcal{A}_{k-1}\right)\left(T^{n} x\right)\right|^{2}\right.\right.\right. \\
& \left.\left.\cdot\left|\left(g_{1} \cdot \overline{g_{1} \circ S_{1}^{h}}\right)\left(S_{1}^{n} y_{1}\right)\right|^{2} \cdots\left|\left(g_{k-1} \cdot \overline{g_{k-1} \circ S_{k-1}^{h}}\right)\left(S_{k-1}^{n} y_{k-1}\right)\right|^{2}\right)^{\frac{1}{2}}\right) \\
\leq & \limsup _{N} C\left(\frac{1}{H}+\frac{\left\|g_{1}\right\|_{\infty}^{2} \cdots\left\|g_{k-1}\right\|_{\infty}^{2}}{H} .\right. \\
& \sum_{h=1}^{H}\left(\frac{1}{N} \sum_{n=1}^{N-h} \mid\left(\left.\mathbb{E}\left(f \cdot \overline{f \circ T^{h}} \mid \mathcal{A}_{k-1}\right)\left(T^{n} x\right)\right|^{2}\right)^{\frac{1}{2}}\right)
\end{aligned}
$$

Prepared using etds.cls 
By Birkhoff's Pointwise Ergodic Theorem we know that there is a set of full measure $X_{k-1}$ on which for each $h$ the average over $n$ in the above inequality converges to

$$
\int \mid\left(\left.\mathbb{E}\left(f \cdot \overline{f \circ T^{h}} \mid \mathcal{A}_{k-1}\right)\right|^{2} d \mu=\left\|\mathbb{E}\left(f \cdot \overline{f \circ T^{h}} \mid \mathcal{A}_{k-1}\right)\right\|_{2}^{2} .\right.
$$

Therefore on the set of full measure $X_{f}=\widehat{X_{f}} \cap X_{k-1}$

$$
\begin{aligned}
\limsup _{N} B_{N} & \leq \frac{C}{H}+\frac{C}{H} \sum_{h=1}^{H}\left\|\mathbb{E}\left(f \cdot \overline{f \circ T^{h}} \mid \mathcal{A}_{k-1}\right)\right\|_{2} \\
& \leq C \lim _{H}\left(\frac{1}{H}+\left(\frac{1}{H} \sum_{h=1}^{H}\left\|\mathbb{E}\left(f \cdot \overline{f \circ T^{h}} \mid \mathcal{A}_{k-1}\right)\right\|_{2}^{2}\right)^{\frac{1}{2}}\right) \\
& =C \cdot N_{k}(f)^{2} .
\end{aligned}
$$

As functions $f$ in $\mathcal{A}_{k}^{\perp}$ are characterized by the property that $N_{k}(f)=0$, Lemma 5 implies that when $f$ is an element of $L^{\infty}(\mu) \bigcap \mathcal{A}_{k}^{\perp}$ we have

$$
\limsup _{N} B_{N}=0
$$

on the set of full measure $X_{f}=\widehat{X_{f}} \bigcap X_{k-1}$. Therefore

$$
\sup _{\epsilon}\left|\frac{1}{N} \sum_{n=1}^{N} f\left(T^{n} x\right) g_{1}\left(S_{1}^{n} y_{1}\right) \cdots g_{k-1}\left(S_{k-1}^{n} y_{k-1}\right) e^{2 \pi i n \epsilon}\right|
$$

converges to $0 \mu$-a.e.. Hence by an application of Lemma 2 we know that for any other dynamical system $\left(Y_{k}, \mathcal{G}_{k}, S_{k}, \nu_{k}\right)$ and any $g \in L^{\infty}\left(\nu_{k}\right)$

$$
\frac{1}{N} \sum_{n=1}^{N} f\left(T^{n} x\right) g_{1}\left(S_{1}^{n} y_{1}\right) \cdots g_{k}\left(S_{k}^{n} y_{k}\right)
$$

converges to 0 in $L^{2}\left(\nu_{k}\right)$. As pointwise convergence of the average in Equation (6) follows from Theorem 2 we have

$$
\frac{1}{N} \sum_{n=1}^{N} f\left(T^{n} x\right) g_{1}\left(S_{1}^{n} y_{1}\right) \cdots g_{k}\left(S_{k}^{n} y_{k}\right)
$$

Prepared using etds.cls 
converges to $0 \nu_{k}$-a.e., when $f$ is in $L^{\infty}(\mu) \bigcap \mathcal{A}_{k}^{\perp}$. Therefore for all $f \in L^{\infty}(\mu)$ we have

$$
\frac{1}{N} \sum_{n=1}^{N}\left[f\left(T^{n} x\right)-\mathbb{E}\left(f \mid \mathcal{A}_{k}\right)\left(T^{n} x\right)\right] g_{1}\left(S_{1}^{n} y_{1}\right) g_{2}\left(S_{2}^{n} y_{2}\right) \cdots g_{k}\left(S_{k}^{n} y_{k}\right)
$$

converges to $0 \nu_{k}$-a.e.. Thus, we have shown that the factors $\mathcal{A}_{k}$ are pointwise characteristic for the multiple term return times averages.

To finish the proof of the theorem it remains to show that

$$
\underset{N}{\limsup }\left|\frac{1}{N} \sum_{n=1}^{N} f\left(T^{n} x\right) g_{1}\left(S_{1}^{n} y\right) g_{2}\left(S_{2}^{n} y_{2}\right) \cdots g_{k}\left(S_{k}^{n} y_{k}\right)\right|^{2} \leq C N_{k+1}(f)^{2}
$$

We use the property just established that the factors $\mathcal{A}_{k}$ are pointwise characteristic for the multiple term return times averages of $k+1$ functions including the arbitrary function $f$ and the Van der Corput lemma. We apply this characteristic property to each of the functions $f \cdot f \circ T^{h}$ and apply the Cauchy Schwartz inequality to obtain our result.

We have

$$
\begin{gathered}
\limsup _{N}\left|\frac{1}{N} \sum_{n=1}^{N} f\left(T^{n} x\right) g_{1}\left(S_{1}^{n} y\right) g_{2}\left(S_{2}^{n} y_{2}\right) \cdots g_{k}\left(S_{k}^{n} y_{k}\right)\right|^{2} \leq \\
C\left[\frac{1}{H}+\frac{1}{H} \sum_{h=1}^{H} \limsup _{N} \mid \frac{1}{N} \sum_{n=1}^{N-h} f\left(T^{n} x\right) f\left(T^{n+h} x\right) .\right. \\
C\left[\frac{1}{H}+\frac{1}{H} \sum_{h=1}^{H} \limsup _{N} \mid \frac{1}{N} \sum_{n=1}^{N-h} \mathbb{E}\left(f \cdot f\left(T_{1}^{n} y_{1}\right)\left|\mathcal{A}_{k}\left(S_{1}^{n+h} y_{1}\right) \cdots g_{k}\left(S_{k}^{n} y_{k}\right) g_{k}\left(S_{k}^{n+h} y_{k}\right)\right|\right]=\right. \\
\left.g_{1}\left(S_{1}^{n} y_{1}\right) g_{1}\left(S_{1}^{n+h} y_{1}\right) \cdots g_{k}\left(S_{k}^{n} y_{k}\right) g_{k}\left(S_{k}^{n+h} y_{k}\right) \mid\right]
\end{gathered}
$$

Applying the characteristic property to each of the functions $f \cdot f \circ T^{h}$ the above inequality is

Prepared using etds.cls 


$$
\leq C\left[\frac{1}{H}+\frac{1}{H} \sum_{h=1}^{H}\left(\left.\limsup _{N}\left|\frac{1}{N} \sum_{n=1}^{N-h}\right| \mathbb{E}\left(f \cdot f\left(T^{h}\right) \mid \mathcal{A}_{k}\right)\left(T^{n} x\right)\right|^{2} \mid\right)^{1 / 2}\right]
$$

because $\left\|g_{i}\right\|_{\infty} \leq 1$.

By Birkhoff's pointwise ergodic theorem and the ergodicity of $T$, the inequality in (17) is

$$
\leq C\left[\frac{1}{H}+\frac{1}{H} \sum_{h=1}^{H}\left\|\mathbb{E}\left(f \cdot f\left(T^{h}\right) \mid \mathcal{A}_{k}\right)\right\|\right] .
$$

Using the Cauchy Schwartz Inequality we obtain that the inequality in (8) is less than or equal to

$$
C\left[\frac{1}{H}+\left(\frac{1}{H} \sum_{h=1}^{H}\left\|\mathbb{E}\left(f \cdot f\left(T^{h}\right) \mid \mathcal{A}_{k}\right)\right\|^{2}\right)^{1 / 2}\right] .
$$

Taking the limit with $H$ gives us the upper bound $C N_{k+1}(f)^{2}$.

3. The $\mathcal{Z}_{k}$ factors are pointwise characteristic for the multiterm return times averages

As noted above, the factors $\mathcal{Z}_{k}$ are smaller than the $\mathcal{A}_{k}$ factors and thus their orthogonal complements $\mathcal{Z}_{k}^{\perp}$ are bigger. Therefore Theorem 4 which we are proving in this section, is an extension of Theorem 3. We will prove Theorem 4 directly from the properties of the factors $\mathcal{Z}_{k}$. The Host-Kra-Ziegler factors, $\mathcal{Z}_{k}$, were defined in 13 by seminorms as follows.

Definition:

- The factor $\mathcal{Z}_{0}$ is equal to the trivial $\sigma$-algebra.

Prepared using etds.cls 
- The factor $\mathcal{Z}_{1}$ can be characterized by the seminorms $\||f|\|_{2}$ where

$$
\||f|\|_{2}^{4}=\lim _{H} \frac{1}{H} \sum_{h=1}^{H}\left|\int f \cdot f \circ T^{h} d \mu\right|^{2}
$$

- The factor $\mathcal{Z}_{2}$ is the Conze-Lesigne factor, $\mathcal{C} \mathcal{L}$. Functions in this factor are characterized by the seminorm ||$\cdot \mid \|_{3}$ such that

$$
\||f|\|_{3}^{8}=\lim _{H} \frac{1}{H} \sum_{h=1}^{H}\left\|\left|f \cdot f \circ T^{h}\right|\right\|_{2}^{4} .
$$

A function $f \in \mathcal{C} \mathcal{L}^{\perp}$ if and only $\||f|\|_{3}=0$.

- More generally B. Host and B. Kra showed in [13 that for each positive integer $k$ we have

$$
\||f|\|_{k+1}^{2 k+1}=\lim _{H} \frac{1}{H} \sum_{h=1}^{H}\left\|\left|f \cdot f \circ T^{h}\right|\right\|_{k}^{2^{k}},
$$

with the condition that $f \in \mathcal{Z}_{k-1}^{\perp}$ if and only if $\||f|\|_{k}=0$.

One can compare the factors $\mathcal{Z}_{k}$ and $\mathcal{A}_{k}$. First, the factors $\mathcal{A}_{k}$ are bigger than the factors $\mathcal{Z}_{k}$. More precisely we have the following.

- The factors $\mathcal{A}_{0}$ and $\mathcal{Z}_{0}$ are equal to the trivial $\sigma$-algebra.

- The factors $\mathcal{A}_{1}$ and $\mathcal{Z}_{1}$ are also identical. The seminorm $\||f|\|_{2}$ and $N_{2}(f)$ are equal. Indeed

$$
\||f|\|_{2}^{4}=\lim _{H} \frac{1}{H} \sum_{h=1}^{H}\left|\int f \cdot f \circ T^{h} d \mu\right|^{2}=N_{2}(f)^{4} .
$$

- The difference starts with the factors $\mathcal{A}_{2}$ and $\mathcal{Z}_{2}$. It is not difficult to find examples where $\mathcal{A}_{2} \neq \mathcal{Z}_{2}$. On the two torus the transformation $(x, y) \rightarrow(x+\alpha, y+\sqrt{\{x\}})$ where $\{x\}$ denotes the fractional part of $x$, is an 
example for which the two factors differ. More generally it can be shown that if the transformation on the two torus is given by $(x, y) \rightarrow(x+\alpha, y+\rho(x))$, where $\rho: \mathbb{T} \rightarrow \mathbb{T}$ is measurable, then $\mathcal{A}_{2}$ always coincides with the full algebra (i.e., the system is 2 -step distal), and $\mathcal{Z}_{2}=\mathcal{A}_{2}$ only when $\rho$ is cohomologous to the affine co-cycle.

Note that the factors $\mathcal{Z}_{k}$ have a very rigid algebraic structure. They have the structure of a pro-nil system. See $\mathbf{1 3}$ for more details on the structure of these factors.

Our induction argument comes from reducing the return times averages by looking at an associated Wiener-Wintner type average using the following lemma.

Lemma 6. Let $(X, \mathcal{F}, \mu, T)$ be an ergodic dynamical system and $f \in L^{\infty}(\mu)$. Then for all positive integers $H$ we have

$$
\underset{N}{\limsup \sup _{t}}\left|\frac{1}{N} \sum_{n=1}^{N} f\left(T^{n} x\right) e^{2 \pi i n t}\right|^{2} \leq C\left(\frac{1}{H}+\frac{1}{H} \sum_{h=1}^{H}\left|\int f \cdot f \circ T^{h} d \mu\right|\right)
$$

where $C$ is an absolute constant derived from the application of the Van der Corput lemma. In particular we have for $\mu$-a.e. $x$

$$
\underset{N}{\limsup \sup _{t}}\left|\frac{1}{N} \sum_{n=1}^{N} f\left(T^{n} x\right) e^{2 \pi i n t}\right|^{2} \leq C\||f|\|_{2}^{2} .
$$

Proof: This is Lemma 2 from the paper [4].

Using this result, we can deduce the following lemma concerning the integral of the limsup of our averages.

Lemma 7. Given $(X, \mathcal{F}, \mu, T)$ an ergodic measure preserving system on a probability measure space and $f \in L^{\infty}$. Then we can find a set of full measure 
$X_{f}$ such that for every $x \in X_{f}$ for each measure preserving dynamical system $\Gamma_{1}=\left(Y_{1}, \mathcal{G}_{1}, \nu_{1}, S_{1}\right)$ and each $g_{1} \in L^{\infty}\left(\nu_{1}\right)$ with $\left\|g_{1}\right\|_{\infty} \leq 1$ we have

$$
\int \limsup _{N} F_{N}^{1}\left(y_{1}\right) d \nu_{1} \leq C\left|\||f|\|_{2}^{2}\right.
$$

where $C$ is an absolute constant derived from the application of the Van der Corput lemma and

$$
F_{N}^{1}\left(y_{1}\right):=\left|\frac{1}{N} \sum_{n=1}^{N} f\left(T^{n} x\right) g_{1}\left(S_{1}^{n} y_{1}\right)\right|^{2} .
$$

Proof: By the BFKO 9] proof of the Return Times Theorem we have pointwise convergence of the above averages, therefore the lim sup on the left hand side of the above expression becomes a limit. Therefore, we have

$$
\begin{aligned}
\int \limsup _{N} F_{N}^{1}\left(y_{1}\right) d \nu_{1} & =\lim _{N} \int F_{N}^{1}\left(y_{1}\right) d \nu_{1} \\
& =\lim _{N} \int\left|\frac{1}{N} \sum_{n=1}^{N} f\left(T^{n} x\right) e^{2 \pi i n t}\right|^{2} d \sigma_{g_{1}}(t)
\end{aligned}
$$

where $\sigma_{g_{1}}$ is the spectral measure associated to $g_{1}$ with respect to the dynamical system $\Gamma_{1}$. Thus

$$
\int \underset{N}{\limsup } F_{N}^{1}\left(y_{1}\right) d \nu_{1} \leq \underset{N}{\limsup \sup _{t}}\left|\frac{1}{N} \sum_{n=1}^{N} f\left(T^{n} x\right) e^{2 \pi i n t}\right|^{2}\left\|g_{1}\right\|_{2}^{2} .
$$

As $\left\|g_{1}\right\|_{\infty} \leq 1$, using Lemma 6 we derive the inequality

$$
\int \underset{N}{\limsup } F_{N}^{1}\left(y_{1}\right) d \nu_{1} \leq C\|\| f \|_{2}^{2}
$$

From Lemma 7 the iteration process follows. For instance, we can use this lemma to prove the following Wiener-Wintner return times result which refines the one obtained in [5].

Prepared using etds.cls 
Lemma 8. Let $(X, \mathcal{F}, \mu, T)$ be an ergodic measure preserving system on a probability measure space and $f \in L^{\infty}(\mu)$. Then for $\mu$-a.e. $x \in X$ for every measure preserving system $\Gamma_{1}=\left(Y_{1}, \mathcal{G}_{\infty}, \nu_{1}, S_{1}\right)$ and each $g_{1} \in L^{\infty}\left(\nu_{1}\right)$ with $\left\|g_{1}\right\|_{\infty} \leq 1$ we have

$$
\int \underset{N}{\lim \sup _{\sup }}{ }_{t}\left|\frac{1}{N} \sum_{n=1}^{N} f\left(T^{n} x\right) g_{1}\left(S_{1}^{n} y_{1}\right) e^{2 \pi i n t}\right|^{2} d \nu_{1} \leq C\||f|\|_{3}^{2}
$$

where $C$ is the absolute constant from the application of the Van der Corput lemma. In particular, for $f \in \mathcal{C L}^{\perp}$ (or equivalently $\||f|\|_{3}=0$ ) we have for $\nu_{1}$-a.e. $y_{1}$

$$
\underset{N}{\limsup \sup _{t}}\left|\frac{1}{N} \sum_{n=1}^{N} f\left(T^{n} x\right) g_{1}\left(S_{1}^{n} y_{1}\right) e^{2 \pi i n t}\right|=0 .
$$

Proof: By the Van der Corput lemma [14] we have

$$
\begin{gathered}
\int \underset{N}{\limsup \sup _{t}}\left|\frac{1}{N} \sum_{n=1}^{N} f\left(T^{n} x\right) g_{1}\left(S_{1}^{n} y_{1}\right) e^{2 \pi i n t}\right|^{2} d \nu_{1} \leq \\
C\left(\frac{1}{H}+\frac{1}{H} \sum_{h=1}^{H} \int \limsup _{N} \mid \frac{1}{N} \sum_{n=1}^{N} f\left(T^{n} x\right) f\left(T^{n+h} x\right) .\right. \\
\left.g_{1}\left(S_{1}^{n} y_{1}\right) g_{1}\left(S_{1}^{n+h} y_{1}\right) \mid d \nu_{1}\right)
\end{gathered}
$$

Using the Cauchy-Schwarz inequality we have that the expression in (12) is less than or equal to

$$
\begin{array}{r}
C\left(\frac{1}{H}+\frac{1}{H} \sum_{h=1}^{H}\left(\int \underset{N}{\limsup } \mid \frac{1}{N} \sum_{n=1}^{N} f\left(T^{n} x\right) f\left(T^{n+h} x\right) .\right.\right. \\
\left.\left.\left.g_{1}\left(S_{1}^{n} y_{1}\right) g_{1}\left(S_{1}^{n+h} y_{1}\right)\right|^{2} d \nu_{1}\right)^{1 / 2}\right)
\end{array}
$$

Similarly to the proof of Lemma 7 as pointwise convergence of the averages holds by Theorem 2 we can rewrite the above $\lim \sup _{N}$ as a $\lim _{N}$ and use the spectral theorem to rewrite the integral in (13) as

$$
\lim _{N} \int\left|\frac{1}{N} \sum_{n=1}^{N} f\left(T^{n} x\right) f\left(T^{n+h} x\right) e^{2 \pi i n t}\right|^{2} d \sigma_{g_{1} \cdot g_{1} \circ S_{1}^{h}}(t) .
$$

Prepared using etds.cls 
Thus by Lemma 6 the expression in 13 is

$$
\begin{aligned}
& \leq C\left(\frac{1}{H}+\frac{1}{H} \sum_{h=1}^{H}\left\|\left|f \cdot f \circ T^{h}\right|\right\|_{2}\left\|g_{1} \cdot g_{1} \circ S_{1}^{h} \mid\right\|_{2}\right) \\
& \leq C\left(\frac{1}{H}+\frac{1}{H} \sum_{h=1}^{H}\left\|\left|f \cdot f \circ T^{h}\right|\right\|_{2}\left\|g_{1}\right\|_{\infty}^{2}\right) \\
& \leq C\left(\frac{1}{H}+\frac{1}{H} \sum_{h=1}^{H}\left\|\mid f \cdot f \circ T^{h}\right\|_{2}\right)
\end{aligned}
$$

on a set of full measure depending only on $f$ as $\|g\|_{\infty} \leq 1$. This set of full measure is, in fact, the intersection of the sets of full measure obtained by the BFKO $\mathbf{9}$ proof of the Return Times Theorem for each function $f \cdot f \circ T^{h}$.

Continuing from above and using Hölder's Inequality we have

$$
\begin{gathered}
\int \limsup _{N} \sup _{t}\left|\frac{1}{N} \sum_{n=1}^{N} f\left(T^{n} x\right) g_{1}\left(S_{1}^{n} y_{1}\right) e^{2 \pi i n t}\right|^{2} d \nu_{1} \leq \\
C\left(\frac{1}{H}+\left(\frac{1}{H} \sum_{h=1}^{H}\left\|\left|f \cdot f \circ T^{h}\right|\right\|_{2}^{4}\right)^{1 / 4}\right) .
\end{gathered}
$$

As the seminorm is defined by

$$
\||f|\|_{3}^{8}=\lim _{H} \frac{1}{H} \sum_{h=1}^{H}\left\|\left|f \cdot f \circ T^{h}\right|\right\|_{2}^{4}
$$

taking the limit on $H$ in the above expression gives

$$
\begin{aligned}
\int \underset{N}{\lim \sup _{\sup }\left|\frac{1}{N} \sum_{n=1}^{N} f\left(T^{n} x\right) g_{1}\left(S_{1}^{n} y_{1}\right) e^{2 \pi i n t}\right|^{2} d \nu_{1}} & \leq C\left(\||f|\|_{3}^{8}\right)^{1 / 4} \\
& =C\||f|\|_{3}^{2} .
\end{aligned}
$$

This proves (10) of Lemma 8. Equation (11) follows directly from the characterization of the $\mathcal{C} \mathcal{L}$ factor.

The induction assumption giving the result on the pointwise characteristic factors for the $\mathcal{Z}_{k}$ factors can now be made. To end it at the $\mathcal{C} \mathcal{L}=\mathcal{Z}_{2}$ level we prove the next lemma.

Prepared using etds.cls 
Lemma 9. Let $(X, \mathcal{F}, \mu, T)$ be an ergodic measure preserving system on a probability measure space. The factor $\mathcal{Z}_{2}$, the Conze Lesigne factor, is pointwise characteristic for the three term return times theorem.

Proof: We denote by $F_{N}\left(y_{1}, y_{2}\right)$ the three term averages with our original function $f$, the fixed system $\Gamma_{1}=\left(Y_{1}, \mathcal{G}_{1}, \nu_{1}, S_{1}\right)$ and the variable one $\Gamma_{2}=\left(Y_{2}, \mathcal{G}_{2}, \nu_{2}, S_{2}\right)$. More precisely we have

$$
F_{N}^{2}\left(y_{1}, y_{2}\right)=\left|\frac{1}{N} \sum_{n=1}^{N} f\left(T^{n} x\right) g_{1}\left(S_{1}^{n} y_{1}\right) g_{2}\left(S_{2}^{n} y_{2}\right)\right|^{2}
$$

By Theorem 2 we have a set of full measure $Y_{g_{1}} \subset Y_{1}$ on which the pointwise convergence of the return times averages with three terms holds for any choice of measure preserving dynamical system $\Gamma_{2}=\left(Y_{2}, \mathcal{G}_{2}, \nu_{2}, S_{2}\right)$ and $g_{2} \in L^{\infty}\left(\nu_{2}\right)$ with $\left\|g_{2}\right\|_{\infty} \leq 1$. Therefore for $y_{1} \in Y_{g_{1}}$ we have

$$
\int \limsup _{N} F_{N}^{2}\left(y_{1}, y_{2}\right) d \nu_{2}=\lim _{N} \int F_{N}^{2}\left(y_{1}, y_{2}\right) d \nu_{2}
$$

Using the spectral measure as before, we can continue from (14)

$$
\begin{aligned}
\int \limsup _{N} F_{N}^{2}\left(y_{1}, y_{2}\right) d \nu_{2} & =\lim _{N} \int\left|\frac{1}{N} \sum_{n=1}^{N} f\left(T^{n} x\right) g_{1}\left(S_{1}^{n} y_{1}\right) e^{2 \pi i n t}\right|^{2} d \sigma_{g_{2}}(t) \\
& \leq{\limsup \sup _{t}}_{N}\left|\frac{1}{N} \sum_{n=1}^{N} f\left(T^{n} x\right) g_{1}\left(S_{1}^{n} y_{1}\right) e^{2 \pi i n t}\right|^{2}\left\|g_{2}\right\|_{\infty}^{2} \\
& \leq{\limsup \sup _{t}}_{N}\left|\frac{1}{N} \sum_{n=1}^{N} f\left(T^{n} x\right) g_{1}\left(S_{1}^{n} y_{1}\right) e^{2 \pi i n t}\right|^{2}
\end{aligned}
$$

as $\left\|g_{2}\right\|_{\infty} \mid \leq 1$. Note that this upper bound is now independent of the choice of $\Gamma_{2}$ and $g_{2}$ so in fact we have

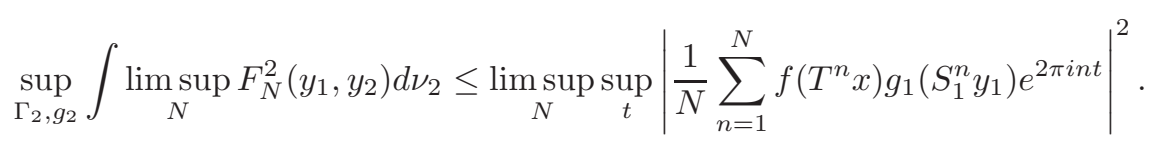

Prepared using etds.cls 
Note that the left hand side of this last inequality is not necessarily measurable. However one can conclude by making the following observation. By Lemma 8 we have

$$
\int \underset{N}{\limsup \sup _{t}}\left|\frac{1}{N} \sum_{n=1}^{N} f\left(T^{n} x\right) g_{1}\left(S_{1}^{n} y_{1}\right) e^{2 \pi i n t}\right|^{2} d \nu_{1} \leq C|||f| \|_{3}^{2} .
$$

Therefore, for $f \in \mathcal{Z}_{2}^{\perp}=\mathcal{C} \mathcal{L}^{\perp}$ (i.e. $\left.\||f|\|_{3}=0\right)$ ) there exists a set of full measure in $Y_{1}$ on which we have

$$
\underset{N}{\limsup \sup _{t}}\left|\frac{1}{N} \sum_{n=1}^{N} f\left(T^{n} x\right) g_{1}\left(S_{1}^{n} y_{1}\right) e^{2 \pi i n t}\right|=0 .
$$

For $y_{1} \in Y_{1}$ we have

$$
\int \underset{N}{\limsup }\left|\frac{1}{N} \sum_{n=1}^{N} f\left(T^{n} x\right) g_{1}\left(S_{1}^{n} y_{1}\right) g_{2}\left(S_{2}^{n} y_{2}\right)\right|^{2} d \nu_{2}=0
$$

for any choice of dynamical system $\Gamma_{2}$ and $g_{2} \in L^{\infty}\left(\nu_{2}\right)$ with $\left\|g_{2}\right\|_{\infty} \leq 1$. Therefore $\mathcal{Z}_{2}$ is pointwise characteristic for the three term return times averages.

We now have the tools necessary to prove our second main result, Theorem 4 , that the $\mathcal{Z}_{k}$ averages are pointwise characteristic for the multiterm return times averages.

Proof: It remains to finish the induction argument which we have started in the above lemmas. Suppose that we know that the $\mathcal{Z}_{j}$ are pointwise characteristic for the $j$-th return times averages and that the bound in (10) from Lemma 8 holds for $j$-th averages for $1 \leq j<k$.

Specifically for $\mu$-a.e. $x \in X$, for every measure preserving system $\Gamma_{j}=$ $\left(Y_{j}, \mathcal{G}_{j}, \nu_{j}, S_{j}\right)$ and each $g_{j} \in L^{\infty}\left(\nu_{j}\right)$ with $\left\|g_{j}\right\|_{\infty} \leq 1$ with $1 \leq j<k-1$ we 
have for $f \in \mathcal{Z}_{k}^{\perp}$

$$
\limsup _{N} \Delta_{N}^{k-1}\left(y_{1}, \ldots, y_{k-1}\right)=0
$$

where

$$
\Delta_{N}^{k-1}\left(y_{1}, \ldots, y_{k-1}\right)=\sup _{t}\left|\frac{1}{N} \sum_{n=1}^{N} f\left(T^{n} x\right) g_{1}\left(S_{1}^{n} y_{1}\right) \cdots g_{k-1}\left(S_{k-1}^{n} y_{k-1}\right) e^{2 \pi i n t}\right|^{2}
$$

and $\Gamma_{k-1}=\left(Y_{k-1}, \mathcal{G}_{k-1}, \nu_{k-1}, S_{k-1}\right)$ is any measure preserving dynamical system and $g_{k-1} \in L^{\infty}\left(\nu_{k-1}\right)$ with $\left\|g_{k-1}\right\|_{\infty} \leq 1$.

By Theorem 2, we know that for any $f \in L^{\infty}(\mu)$ and $1 \leq j<k$ we can find sets $X_{f}$ of full measure such that if $x \in X_{f}$, then for any other dynamical system $\Gamma_{1}=\left(Y_{1}, \mathcal{G}_{1}, S_{1}, \nu_{1}\right)$ and any $g_{1} \in L^{\infty}\left(\nu_{1}\right)$ with $\left\|g_{1}\right\|_{\infty} \leq 1$, there exists a set of full measure $Y_{g_{1}}$ such that for each $y_{1}$ in $Y_{g_{1}}$ then $\ldots$ for any other dynamical system $\Gamma_{k-1}=\left(Y_{k-1}, \mathcal{G}_{k-1}, S_{k-1}, \nu_{k-1}\right)$ and any $g_{k-1} \in L^{\infty}\left(\nu_{k-1}\right)$ with $\left\|g_{k-1}\right\|_{\infty} \leq 1$ there exist a set of full measure $Y_{g_{k-1}}$ in $Y_{k-1}$ such that if $y_{k-1} \in Y_{g_{k-1}}$ we have the pointwise convergence of the return times averages with $k$ terms for any other dynamical system $\Gamma_{k}=\left(Y_{k}, \mathcal{G}_{k}, S_{k}, \nu_{k}\right)$ and any $g_{j} \in L^{\infty}\left(\nu_{j}\right)$ with $\left\|g_{j}\right\|_{\infty} \leq 1$.

Thus for $x, y_{1}, \ldots y_{k-1}$ as above we have

$$
\int \limsup _{N} F_{N}^{k}\left(y_{1}, \ldots, y_{k}\right) d \nu_{k}=\lim _{N} \int F_{N}^{k}\left(y_{1}, \ldots, y_{k}\right) d \nu_{k}
$$

where

$$
F_{N}^{k}\left(y_{1}, \ldots, y_{k}\right)=\left|\frac{1}{N} \sum_{n=1}^{N} f\left(T^{n} x\right) g_{1}\left(S_{1}^{n} y_{1}\right) \ldots g_{k}\left(S_{k}^{n} y_{k}\right)\right|^{2} .
$$

We would like to show that $\mathcal{Z}_{k}$ is pointwise characteristic for the $k$-return times averages and that we have for $f \in \mathcal{Z}_{k+1}^{\perp}$

$$
\limsup _{N} \Delta_{N}^{k}\left(y_{1}, \ldots, y_{k}\right)=0
$$

Prepared using etds.cls 
where the

$$
\Delta_{N}^{k}\left(y_{1}, \ldots, y_{k}\right)=\sup _{t}\left|\frac{1}{N} \sum_{n=1}^{N} f\left(T^{n} x\right) g_{1}\left(S_{1}^{n} y_{1}\right) \cdots g_{k}\left(S_{k}^{n} y_{k}\right) e^{2 \pi i n t}\right|^{2}
$$

$\Gamma_{k}$ and $g_{k}$ are as defined above. This will complete our proof by induction.

Using the spectral theorem and continuing from (16) we have

$$
\begin{array}{r}
\int \limsup _{N} F_{N}^{k}\left(y_{1}, \ldots, y_{k}\right) d \nu_{k}= \\
\lim _{N} \int\left|\frac{1}{N} \sum_{n=1}^{N} f\left(T^{n} x\right) g_{1}\left(S_{1}^{n} y_{1}\right) \cdots g_{k-1}\left(S_{k-1}^{n} y_{k-1}\right) e^{2 \pi i n t}\right|^{2} d \sigma_{g_{k}}(t) \leq \\
{\limsup \sup _{t}}_{t}\left|\frac{1}{N} \sum_{n=1}^{N} f\left(T^{n} x\right) g_{1}\left(S_{1}^{n} y_{1}\right) \cdots g_{k-1}\left(S_{k-1}^{n} y_{k-1}\right) e^{2 \pi i n t}\right|^{2}\left\|g_{k}\right\|_{\infty} \leq \\
\limsup _{N} \sup _{t}\left|\frac{1}{N} \sum_{n=1}^{N} f\left(T^{n} x\right) g_{1}\left(S_{1}^{n} y_{1}\right) \cdots g_{k-1}\left(S_{k-1}^{n} y_{k-1}\right) e^{2 \pi i n t}\right|^{2}
\end{array}
$$

as $\left\|g_{k}\right\| \leq 1$.

Note that this upper bound is now independent of the choice of $\Gamma_{k}$ and $g_{k}$ so

$$
\begin{gathered}
\sup _{\Gamma_{k}, g_{k}} \int \limsup _{N} F_{N}^{k}\left(y_{1}, \ldots, y_{k}\right) d \nu_{k} \leq \\
{\limsup \sup _{t}}_{\operatorname{lin}_{N}}\left|\frac{1}{N} \sum_{n=1}^{N} f\left(T^{n} x\right) g_{1}\left(S_{1}^{n} y_{1}\right) \cdots g_{k-1}\left(S_{k-1}^{n} y_{k-1}\right) e^{2 \pi i n t}\right|^{2} .
\end{gathered}
$$

In the same manner as shown in Lemma 9, from the equation (15) and (17) one can conclude that $\mathcal{Z}_{k}$ is pointwise characteristic for the $k$-th return times averages.

To complete the induction step it remains to show that (15) holds for $k$.

To make the reading of the induction proof easier we show how one can prove that $\mathcal{Z}_{4}$ is pointwise characteristic for the 5 -th return times averages. The reader will check that the arguments extend without difficulty to arbitrary $k$. So we want to show that if $\||f|\|_{5}=0$ then

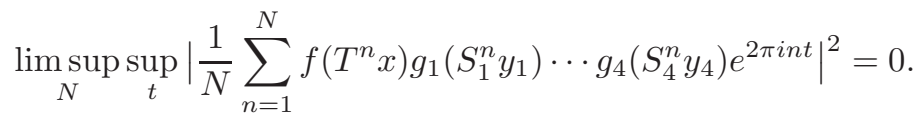

Prepared using etds.cls 
By the Van der Corput lemma, Theorem 2 and Cauchy-Schwartz inequality we have

$$
\begin{aligned}
& \int \underset{N}{\limsup \sup _{t}}\left|\frac{1}{N} \sum_{n=1}^{N} f\left(T^{n} x\right) g_{1}\left(S_{1}^{n} y_{1}\right) \cdots g_{4}\left(S_{4}^{n} y_{4}\right) e^{2 \pi i n t}\right|^{2} d \nu_{4} \\
\leq & C\left(\frac{1}{H_{1}}+\left(\frac{1}{H_{1}} \sum_{h_{1}=1}^{H_{1}} \limsup _{N} \int \mid \frac{1}{N} \sum_{n=1}^{N} f\left(T^{n} x\right) f\left(T^{n+h_{1}} x\right) .\right.\right. \\
& \left.\left.\left.\cdots g_{4}\left(S_{4}^{n} y_{4}\right) g_{4}\left(S_{4}^{n+h_{1}} y_{4}\right)\right|^{2} d \nu_{4}\right)^{1 / 2}\right) .
\end{aligned}
$$

By the spectral theorem this last term is equal to

$$
\begin{aligned}
& \left(\frac{1}{H_{1}} \sum_{h_{1}=1}^{H_{1}} \limsup _{N} \int \mid \frac{1}{N} \sum_{n=1}^{N} f\left(T^{n} x\right) f\left(T^{n+h_{1}} x\right) .\right. \\
& \left.\left.\cdots g_{3}\left(S_{3}^{n} y_{3}\right) g_{3}\left(S_{3}^{n+h_{1}} y_{3}\right) e^{2 \pi i n t}\right|^{2} d \sigma_{g_{4} \cdot g_{4} \circ S_{4}^{h_{1}}}\right)^{1 / 2} .
\end{aligned}
$$

As $\left\|g_{4}\right\|_{\infty} \leq 1$, the generic term in this sum is less than

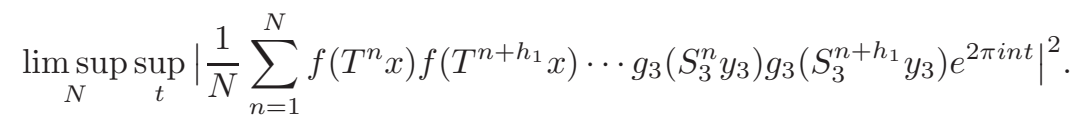

One can conclude that for the appropriate universal sets for $x, y_{1}, y_{2}$, and $y_{3}$ if

$$
\begin{aligned}
& \lim _{H_{1}} \frac{1}{H_{1}} \sum_{h_{1}=1}^{H_{1}} \limsup _{N} \sup _{t} \mid \frac{1}{N} \sum_{n=1}^{N} f\left(T^{n} x\right) f\left(T^{n+h_{1}} x\right) . \\
&\left.\cdots g_{3}\left(S_{3}^{n} y_{3}\right) g_{3}\left(S_{3}^{n+h_{1}} y_{3}\right) e^{2 \pi i n t}\right|^{2}=0
\end{aligned}
$$

then

$$
\int \underset{N}{\lim \sup _{\sup }} \sup _{t}\left|\frac{1}{N} \sum_{n=1}^{N} f\left(T^{n} x\right) g_{1}\left(S_{1}^{n} y_{1}\right) \cdots g_{4}\left(S_{4}^{n} y_{4}\right) e^{2 \pi i n t}\right|^{2} d \nu_{4}=0
$$

for all measure preserving system $\Gamma_{4}=\left(Y_{4}, \mathcal{G}_{4}, \nu_{4}, S_{4}\right)$ and each $g_{4}$ with $\left\|g_{4} \mid\right\|_{\infty} \leq 1$.

We then derive that

$$
\underset{N}{\limsup \sup _{t}}\left|\frac{1}{N} \sum_{n=1}^{N} f\left(T^{n} x\right) g_{1}\left(S_{1}^{n} y_{1}\right) \cdots g_{4}\left(S_{4}^{n} y_{4}\right) e^{2 \pi i n t}\right|^{2}=0
$$

Prepared using etds.cls 
which is the content of (18).

Therefore we look at the quantity

$$
\int \limsup _{N} \sup _{t}\left|\frac{1}{N} \sum_{n=1}^{N} f\left(T^{n} x\right) f\left(T^{n+h_{1}} x\right) \cdots g_{3}\left(S_{3}^{n} y_{3}\right) g_{3}\left(S_{3}^{n+h_{1}} y_{3}\right) e^{2 \pi i n t}\right|^{2} d \nu_{3}
$$

Again by Van der Corput lemma, Theorem 2 and Cauchy Schwartz inequality this quantity is less than

$$
\begin{gathered}
C\left(\frac{1}{H_{2}}+\left(\frac{1}{H_{2}} \sum_{h_{2}=1}^{H_{2}} \limsup _{N} \int \mid \frac{1}{N} \sum_{n=1}^{N} f\left(T^{n} x\right) f\left(T^{n+h_{1}} x\right) f\left(T^{n+h_{2}} x\right) f\left(T^{n+h_{1}+h_{2}} x\right)\right.\right. \\
\left.\left.\left.\cdots g_{3}\left(S_{3}^{n} y_{3}\right) g_{3}\left(S_{3}^{n+h_{1}} y_{3}\right) g_{3}\left(S_{3}^{n+h_{2}} y_{3}\right) g_{3}\left(S_{3}^{n+h_{1}+h_{2}} y_{3}\right)\right|^{2} d \nu_{3}\right)^{1 / 2}\right) .
\end{gathered}
$$

As shown previously, by the spectral theorem this last term is equal to

$$
\begin{array}{r}
\left(\frac{1}{H_{2}} \sum_{h_{2}=1}^{H_{2}} \limsup _{N} \int \mid \frac{1}{N} \sum_{n=1}^{N} f\left(T^{n} x\right) f\left(T^{n+h_{1}} x\right) f\left(T^{n+h_{2}} x\right) f\left(T^{n+h_{1}+h_{2}} x\right)\right. \\
g_{2}\left(S_{2}^{n} y_{2}\right) g_{2}\left(S_{2}^{n+h_{1}} y_{2}\right) g_{2}\left(S_{2}^{n+h_{2}} y_{2}\right) g_{2}\left(S_{2}^{n+h_{1}+h_{2}} y_{2}\right) \\
\left.e^{2 \pi i n t}\right|^{2} d \sigma_{\left.g_{3} \cdot g_{3} \circ S_{3}^{h_{1}} g_{3} \circ S_{3}^{h_{2}} g_{3} \circ S_{3}^{h_{1}+h_{2}}\right)^{1 / 2}} .
\end{array}
$$

This in turn is less than

$$
\begin{array}{r}
\frac{1}{H_{2}} \sum_{h_{2}=1}^{H_{2}} \lim \sup _{N} \sup _{t} \mid \frac{1}{N} \sum_{n=1}^{N} f\left(T^{n} x\right) f\left(T^{n+h_{1}} x\right) f\left(T^{n+h_{2}} x\right) f\left(T^{n+h_{1}+h_{2}} x\right) \\
\left.g_{2}\left(S_{2}^{n} y_{2}\right) g_{2}\left(S_{2}^{n+h_{1}} y_{2}\right) g_{2}\left(S_{2}^{n+h_{2}} y_{2}\right) g_{2}\left(S_{2}^{n+h_{1}+h_{2}} y_{2}\right) e^{2 \pi i n t}\right|^{2}
\end{array}
$$

We integrate this term with respect to $\nu_{2}$. Another application of the Van der Corput lemma, Theorem 2, Cauchy Schwartz Inequality and the spectral theorem leads to the estimate

$$
\left(\frac{1}{H_{3}} \sum_{h_{3}=1}^{H_{3}} \limsup _{N} \sup _{t} \mid \frac{1}{N} \sum_{n=1}^{N} f\left(T^{n} x\right) f\left(T^{n+h_{1}} x\right) f\left(T^{n+h_{2}} x\right) f\left(T^{n+h_{1}+h_{2}} x\right)\right.
$$

Prepared using etds.cls 


$$
\begin{gathered}
f\left(T^{n+h_{3}} x\right) f\left(T^{n+h_{1}+h_{3}} x\right) f\left(T^{n+h_{2}+h_{3}} x\right) f\left(T^{n+h_{1}+h_{2}+h_{3}} x\right) g_{1}\left(S_{1}^{n} y_{1}\right) g_{1}\left(S_{1}^{n+h_{1}} y_{1}\right) \\
g_{1}\left(S_{1}^{n+h_{2}} y_{1}\right) g_{1}\left(S_{1}^{n+h_{1}+h_{2}} y_{1}\right) g_{1}\left(S_{1}^{n+h_{3}} y_{1}\right) g_{1}\left(S_{1}^{n+h_{1}+h_{3}} y_{1}\right) g_{1}\left(S_{1}^{n+h_{2}+h_{3}} y_{1}\right) \\
\left.\left.g_{1}\left(S_{1}^{n+h_{1}+h_{2}+h_{3}} y_{1}\right) e^{2 \pi i n t}\right|^{2}\right)^{1 / 2} \cdot
\end{gathered}
$$

Finally integrating this last term with respect to $\nu_{1}$ we can use (10) and combine the previous inequalities to get the upper bound

$$
\begin{array}{r}
C\left(\sum_{i=1}^{3} \frac{1}{H_{i}}+\frac{1}{\prod_{i=1}^{3} H_{i}} \sum_{h_{3}=1}^{H_{3}} \sum_{h_{2}=1}^{H_{2}} \sum_{h_{1}=1}^{H_{1}} \| f \cdot f \circ T^{h_{1}} .\right. \\
\left.f \circ T^{h_{2}} f \circ T^{h_{1}+h_{2}} \cdot f \circ T^{h_{3}} f \circ T^{h_{1}+h_{3}} f \circ T^{h_{2}+h_{3}} f \circ T^{h_{1}+h_{2}+h_{3}} \mid \|_{3}\right)
\end{array}
$$

Applying (9) three times gives the upper bound

$$
C\||f|\|_{5}^{8}
$$

Thus if $\||f|\|_{5}=0$ we can go back and step by step obtain universal sets for $x, y_{1}, y_{2}, y_{3}$ for which (18) holds.

\section{Remarks:}

1. In Theorem 5 (see Equation (3)), we proved that we have a pointwise upper bound on the average of multiple terms as follows

$$
\underset{N}{\limsup }\left|\frac{1}{N} \sum_{n=1}^{N} f\left(T^{n} x\right) g_{1}\left(S_{1}^{n} y_{1}\right) \cdots g_{k}\left(S_{k}^{n} y_{k}\right)\right|^{2} \leq C N_{k+1}(f)^{2} .
$$

We asked in a previous version of this paper whether for $k \geq 2$, one can replace in these inequalities the $N_{k}$ seminorms for the $\mathcal{A}_{k}$ with those defining the $\mathcal{Z}_{k}$ factors.

- It was shown in [13] that $\||f|\|_{2} \leq\left\|\mathbb{E}\left(f \mid \mathcal{K}_{T}\right)\right\|_{2}$ but we can not find an absolute constant $C$ for which $\left\|\mathbb{E}\left(f \mid \mathcal{K}_{T}\right)\right\|_{2} \leq C\||f|\|_{2}$. 
- As pointed out by the referee one can not replace the $N_{k}$ seminorms in Equation (3) with those defining the $\mathcal{Z}_{k}$ factors. For $T$ an irrational rotation on the circle the Kronecker factor is $\mathcal{F}$ and $\mathbb{E}\left(f, \mathcal{K}_{T}\right)=f$. Then $\left\|\mathbb{E}\left(f \mid \mathcal{K}_{T}\right)\right\|_{2}=\|\hat{f}(k)\|_{l^{2}(Z)}$. Direct computations show that $\||f|\|_{2}=$ $\|\hat{f}(k)\|_{l^{4}(Z)}$. Therefore one can not find an absolute constant $C$ for which $\|\hat{f}\|_{l^{4}(\mathbb{Z})} \leq C\|\hat{f}\|_{l^{2}(\mathbb{Z})}$. Consider the average $\frac{1}{N} \sum_{n=1}^{N} f\left(T^{n} x\right) \bar{f}\left(T^{n} y\right)$.

Then we have

$$
\lim _{N}\left|\frac{1}{N} \sum_{n=1}^{N} f\left(T^{n} x\right) \bar{f}\left(T^{n} y\right)\right|=\left.\left|\sum_{k \in \mathbb{Z}}\right| \hat{f}(k)\right|^{2} e^{2 \pi i k(x-y)} \mid .
$$

The right hand side of the above inequality is less than $\sum_{k \in \mathbb{Z}}|\hat{f}(k)|^{2}=$ $\left\|\mathbb{E}\left(f \mid \mathcal{K}_{T}\right)\right\|_{2}^{2}$. However one can not find an absolute constant $C$ for which $\left.\left|\sum_{k \in \mathbb{Z}}\right| \hat{f}(k)\right|^{2} e^{2 \pi i k(x-y)} \mid \leq C\|\hat{f}(k)\|_{l^{4}(Z)}^{2}$.

2. The authors of this paper are writing a survey of the Return Times Theorem [6] which will include more details of the historical developments of Theorem 1 and 2 and related questions such as the ones noted above.

Acknowledgements The authors thank the referees for their comments.

\section{REFERENCES}

[1] I. Assani. "Multiterm return time theorem for weakly mixing systems" Annales de L'Institut Henri Poincare,vol 36,2,153-165,(2000).

[2] I. Assani. Wiener Wintner Ergodic Theorems. World Scientific Publishing Co., New Jersey, London, Singapore, Hong Kong, 2003.

Prepared using etds.cls 
[3] I. Assani. "Characteristic factors for some non conventional ergodic averages." Preprint, 2003.

[4] I. Assani. "Pointwise Convergence of Ergodic Averages along Cubes." J. Analyse Math. $110(2010), 241-269$.

[5] I. Assani, E. Lesigne and D. Rudolph. "Wiener Wintner return-times ergodic theorem." Isr. J. Math. 92 (1995) 375-395.

[6] I. Assani and K. Presser. "Survey of the Return Times Theorem." In Progress, 2010.

[7] G. D. Birkhoff. "Proof of the ergodic theorem." Proc. Nat. Acad. Sci. 17 (1931), 656-660.

[8] J. Bourgain. "Return time sequences of dynamical systems." Unpublished Preprint, 1988.

[9] J. Bourgain, H. Furstenberg, Y. Katznelson, and D. Ornstein. Appendix to J. Bourgain: "Pointwise ergodic theorems for arithmetic sets." Inst. Hautes Études Sci. Publ. Math. 69 (1989), 5-45.

[10] H. Furstenberg. "The structure of distal flows." American Journal of Mathematics 85 no. 3 (1963), 477-515.

[11] H. Furstenberg. "Ergodic behavior of diagonal measures and a theorem of Szemerédi on arithmetic progressions." J. Analyse Math. 31 (1977), $204-256$.

[12] H. Furstenberg and B. Weiss. "A mean ergodic theorem for $\frac{1}{N} \sum_{n=1}^{N} f\left(T^{n} x\right) g\left(T^{2 n} x\right)$." Convergence in Ergodic Theory and Probability 5 (1996), 193-227.

[13] B. Host and B. Kra. "Nonconventional ergodic averages and nilmanifolds." Ann. of Math. (2) $161(2005), 397-488$.

[14] L. Kuipers and H. Niederreiter. Uniform distribution of sequences. Wiley-Interscience, John Wiley \& Sons, New York, 1974.

[15] D. Rudolph. "A joinings proof of Bourgain's return time theorem." Ergodic Theory Dynam. Systems14 no. 1 (1994), 197 - 203.

[16] D. Rudolph. "Eigenfunctions of $T \times S$ and the Conze-Lesigne algebra." Ergodic theory and its connections with harmonic analysis. London Math. Soc. Lecture Note Ser. 205 (1995), $369-432$.

[17] D. Rudolph. "Fully generic sequences and a multiple-term return times theorem." Invent. Math. 131 no. 1 (1998), 199-228.

Prepared using etds.cls 
[18] T. Ziegler. "Universal characteristic factors and Furstenberg averages." J. Amer. Math. Soc., 20, (2007), 53-97.

Prepared using etds.cls 